\title{
Complex local features as determinants of pattern discrimination
}

\author{
ROBERT PASNAK and ZITA E. TYER \\ George Mason University, Fairfax, Virginia
}

\begin{abstract}
Random polygons were generated in a manner that permitted us to test whether unfamiliar patterns were discriminated on the basis of their most complex or simplest local features. Results showed that subjects based discrimination primarily on the most complex local features, although the simpler features were not entirely ignored. This tendency persisted, although subjects had ample opportunity to learn that the complex and simple local features provided equally reliable cues for discrimination.
\end{abstract}

Recent investigations have shown that local characteristics of visual patterns may play an important role in discrimination (Foster, 1978; Hoffman, 1980; Kimchi \& Palmer, 1982; Pasnak \& Tyer, 1985). Gibson (1969) anticipated this result, theorizing that a visual pattern might sometimes be discriminated on the basis of distinctive features that make up only a small portion of the pattern's contour. Pasnak (1971) showed that this prediction was more correct for complex patterns than for simple ones. Furthermore, he found an inverse linear relationship between the percentage of complex patterns that contained duplicates of any local feature and the probability that that feature would serve as a discriminative stimulus.

Pasnak and Tyer (1985) extended this idea, reasoning that the most complex sections of any pattern's contour were the least likely to be duplicated by any other pattern's contour and, hence, would be distinctive local features. Their rationale was that a complex section of contour has many degrees of freedom and, thus, has a lower probability of being reproduced by any independent and random process than do simpler contours with fewer degrees of freedom. A discrimination experiment with randomly generated polygons showed that this hypothesis was correct. When contours were of equal length, differences in complex contours were detected much more often than were differences in simple contours. Hence, on brief $(1-\mathrm{sec})$ presentations, polygons were likely to be considered the same if they differed only in a simple contour section (a section divided into only a few line segments). Subjects were much more likely to realize that patterns were different if the difference comprised a complex contour section (one divided into many line segments). This bias held, even though the total amount of contour differentiating the patterns was always constant.

This finding can be extended by contriving patterns so that discriminations can readily be made, by using some

This research was supported by USPHS Grant No. MH 17309 from the National Institute of Mental Health. Reprints can be obtained from either author at the Department of Psychology, George Mason University, Fairfax, VA 22030. local feature other than the most complex one, and presenting the patterns on repeated trials to test the effect of learning. If subjects continue to discriminate between patterns on the basis of complex local features, despite ample opportunity to learn that simple local features are also available, then the tendency to rely on the most complex parts of the patterns for discrimination may be viewed as a general and enduring tendency.

This approach was taken in the present experiment by repeatedly exposing subjects to comparison (Co) patterns that differed from a standard (St) pattern in both complex and simple portions of contour. Since a differentiating local feature (equivalent to that in the complex portions of contour) was presented in a simple portion of contour, subjects might have come to detect it with repeated opportunities, unless they were biased to respond to the complex portion of the pattern. This design made it possible to determine whether subjects sometimes persist in basing pattern discriminations on distinguishing local features found in complex portions of contour, and fail to base discriminations on equally distinctive local features in a less complex section of the contour.

\section{METHOD}

\section{Subjects}

The subjects were 40 experimentally naive undergraduate volunteers.

\begin{abstract}
Apparatus
A Kodak RA950 carousel projector fitted with a Wollensak tachistoscopic shutter was employed in this experiment.

\section{Stimuli}

In an attempt to further test the generality of the findings of Pasnak and Tyer (1985), we constructed the patterns on a different type of grid, using a different definition of complexity. Here, polygons were generated on rectangular grids that were divided into four sections of quadrants-upper and lower, right and left. Relative complexity of the four sections of the polygons' contours was defined as the relative number of line segments in the contour in each quadrant. All line segments had equal lengths. Thus, if the number of line segments in a given quadrant of the visual field is relatively high, that portion of the contour is relatively complex. If, on the other hand, the contour in another quadrant of the visual field contains relatively few line segments, that section of contour is relatively simple. Since complexity is defined in this relative
\end{abstract}


manner, changing the polygon's size or visual angle, as might happen if a viewer moved nearer or farther away, does not change the relative complexity of the various parts of the contour, even though the area of the visual field occupied by that contour changes.

One St polygon was generated with nine line segments in one complex quadrant, three in the simple quadrant, and six in the two intermediate quadrants (see Figure 1). All line segments were 1 in. (25.4 mm) long, and were generated by a sequential random process. The proper numbers of points in each quadrant were selected sequentially from a random numbers table with the restrictions that each be 1 in. from the last selected, and that one point be on the boundary of each quadrant. (This last feature produces a closed polygon for which any quadrant can be replaced without disturbing the remaining quadrants.) The points were sequentially connected with straight lines.

Next, six training Co polygons were generated. These were all identical to the St in the two intermediate quadrants, but all were different in both the complex and simple quadrants. For each $\mathrm{Co}$, one point was randomly changed in both the simple and complex quadrants, thereby changing the line segments (two in each quadrant) defined by those points. A random determination was made of which point would be changed in each case, with the restriction that all points in the complex quadrant be changed equally often. There were fewer points in the simple quadrant, so they had to be changed more often. The absolute amount and configuration of contour changed were always the same (one point, defining two line segments of equal length) for changes in both the simple and complex quadrants. However, a larger percentage of the simple contours would change $(67 \%)$ than would the complex contours $(22 \%)$.

Sixteen test Co patterns were also generated. Eight of these varied from the St only in the complex quadrant, and 8 varied only in the simple quadrant. Changes were made randomly as described above.

\section{Procedure}

Four groups of 10 subjects were run, each on a different orientation of the patterns, in 45-min sessions. Subjects were seated in two frontal rows of 5, at distances of 4.62-5.49 $\mathrm{m}$ from the screen. Projected polygons had maximum extents of $101.6 \mathrm{~cm}$ on this screen, and hence subtended a maximum visual angle of $10^{\circ} 23^{\prime}$. Luminance of the polygons was $445.38 \mathrm{~cd} / \mathrm{m}^{2}$, and luminance of the screen background was $40.26 \mathrm{~cd} / \mathrm{m}^{2}$.

The subjects were told that pairs of random polygons would be presented and that they should write whether the polygons were identical or different on the answer sheets provided. First, a St was presented for $1 \mathrm{sec}$; after $5 \mathrm{sec}$, during which the screen was blank, a Co was presented for $1 \mathrm{sec}$. In the following $5 \mathrm{sec}$, the subjects were to write whether the members of each pair were identical or different, and were required to guess if they were uncertain.

Half of the 64 trials consisted of presentations of a St twice in succession in order to equalize the effectiveness of guessing "different" with that of guessing "identical." The other 32 trials consisted of single pairings of each St with the Co patterns appropriate to it. The order of trials was randomized for each set of subjects.

Standard

2

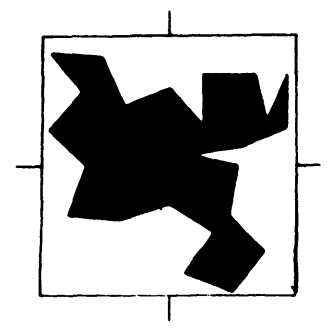

3
Training Co

21

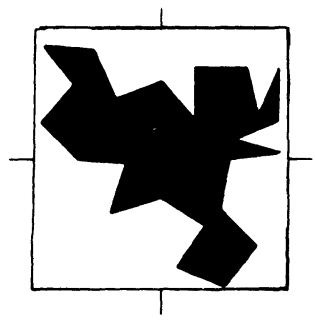

3
Thus, for the first eight trials, eight pairs of polygons were presented in a random order. Four of the pairs consisted of the St presented twice. The other four pairs consisted of pairings of the St with four different Co polygons. Each of these polygons differed from the St in both the complex and simple contour sections. Although the subjects were given no feedback on the correctness of their decisions, they had four opportunities to observe that when polygons differed from the St, they differed in both the simple and complex contour sections.

Next, with no break in the presentation of stimuli, the first test stage began. This stage also consisted of eight randomly ordered pairs of polygons, four of which were the St presented twice. Two of the other pairs consisted of the St paired with test Co polygons, which differed from the St only in the complex contour section. The other two pairs consisted of the St paired with test Co polygons, which differed only in the simple contour section. If subjects were responding primarily to the complex section, they would make more correct responses on the test Co polygons which differed from the St only in the complex section. Conversely, they would make more correct responses on the test Co polygons differing from the St only in the simple section if they had been using the simple section as the discriminative stimulus.

The second stage of training and testing comprised 16 trials, similar to the first 16 trials, except that new Co polygons were used. The third series of 16 trials again involved new Co polygons, as did the fourth and final stage of training and testing.

\section{RESULTS}

The data from the training trials were analyzed via a split-plot factorial ANOVA with orientation of stimulus pattern $\left(0^{\circ}, 90^{\circ}, 180^{\circ}, 270^{\circ}\right)$ as the nonrepeated measure variable and stage of training $(1,2,3,4)$ as the repeated measure variable. Correct discrimination of the St and Co stimuli as "different" was the dependent measure. The main effect of stage of training was significant $[F(3,9)=14.31, p<.01]$ and showed a significant linear trend $[F(1,9)=12.60, p<.01]$. Accuracy reached a level of $86 \%$ during the third stage of exposure and did not improve at all during the last eight trials.

The rotation of pattern variable was not significant $[F(3,9)=.168, p>.05]$. Since this variable was essentially a control and had no effect, the groups were combined for subsequent analyses.

Data from the four test stages (wherein St and Co polygons differed in only one contour section) were analyzed via a randomized block factorial ANOVA. Again, there was significant improvement across stages of training

$$
\text { Simple Test co }
$$

Complex Test co

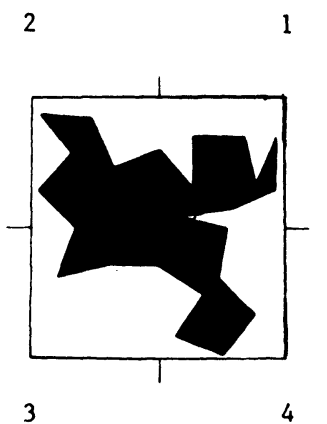

1

2

1

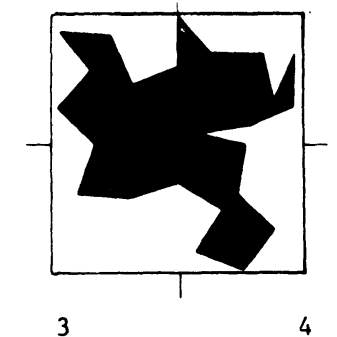

Figure 1. Labels, numerals, and dashes indicating divisions of contour sections were not visible to the subjects. Section 1 is the complex section; Section 3 is the simple section. 
Table 1

Means and Standard Deviations for Correct Responses

\begin{tabular}{|c|c|c|c|c|}
\hline & Stage 1 & Stage 2 & Stage 3 & Stage 4 \\
\hline Pairs & Mean SD & Mean SD & Mean SD & Mean SD \\
\hline St-St & $\begin{array}{r}3.12 \\
.19\end{array}$ & $\begin{array}{r}3.30 \\
.19\end{array}$ & $\begin{array}{r}3.52 \\
.08\end{array}$ & $\begin{array}{r}3.57 \\
.11\end{array}$ \\
\hline \multirow[t]{2}{*}{ St-Co } & $\begin{array}{r}2.97 \\
.11\end{array}$ & $\begin{array}{r}3.15 \\
.23\end{array}$ & $\begin{array}{r}3.37 \\
.08\end{array}$ & $\begin{array}{r}3.30 \\
.12\end{array}$ \\
\hline & $\frac{\text { Test } 1^{*}}{\text { Mean SD }}$ & $\frac{\text { Test } 2}{\text { Mean Sd }}$ & $\frac{\text { Test } 3}{\text { Mean SD }}$ & $\frac{\text { Test } 4}{\text { Mean SD }}$ \\
\hline $\mathrm{St}-\mathrm{St}$ & $\begin{array}{r}3.30 \\
.19\end{array}$ & $\begin{array}{r}3.45 \\
.17\end{array}$ & $\begin{array}{r}3.45 \\
.15\end{array}$ & $\begin{array}{r}3.75 \\
.15\end{array}$ \\
\hline St-Co & $\begin{array}{r}1.60 \\
.59\end{array}$ & $\begin{array}{c}\text { ple Section } \\
2.84 \\
.12\end{array}$ & fers $\begin{array}{r}2.24 \\
.25\end{array}$ & $\begin{array}{r}2.14 \\
.33\end{array}$ \\
\hline \multicolumn{5}{|c|}{ Complex Section Differs } \\
\hline & .37 & .10 & .13 & .18 \\
\hline
\end{tabular}

*Note that Test 1 followed Stage 1, Test 2 followed Stage 2, and so forth. Co polygons on test trials differed from St polygons in only one contour section, rather than two.

$[F(3,9)=4.62, p<.05]$. More importantly, the Co patterns differing from the $\mathrm{St}$ in a section of complex contour were easier to discriminate than those differing in a section of simple contour $[F(1,9)=18.92, p<.01]$. The complexity effect was robust: twice as many incorrect responses were made when test Co patterns differed from the St in the simple section.

Similar analyses indicated that performance improved on St-St pairs during test trials $[F(3,9)=4.32, p<.05]$, and that the rate of improvement was not different from that found on nontest trials $[F(3,9)=1.28, p>.05]$. Also, more correct responses were made on the Co polygons which differed from St in two contour sections than on those which differed in only one contour section $[F(1,3)=10.78, p<.05]$. The lack of a significant interaction indicates that this tendency did not change over trials $[F(3,9)=3.58, p>.05]$. These results are also shown in Table 1.

\section{DISCUSSION}

As was expected, subjects became better able to discriminate the $\mathrm{St}$ from $\mathrm{Co}$, whether the patterns differed in sections of simple contour, in sections of complex contour, or in both. However, if the patterns differed in only one section, subjects were always more accurate when the difference was in the complex section of contour. This finding confirms the result obtained by Pasnak and Tyer (1985), using a different type of polygon. Furthermore, this tendency to employ the complex sections as discriminative stimuli did not diminish, in spite of ample opportunities to learn that simple sections were an equally accurate basis for discrimination. This is not to say that subjects were completely unresponsive to differences in simple contour sections. To the contrary, discriminations were most accurate when Co differed from St in both complex and simple contour regions. Yet, when there was only one differ- ence between patterns, it was much more likely to be detected if it was in a complex section of contour. The formal definitions of complexity used in this experiment and by Pasnak and Tyer (1985) differ, but in both experiments, "complexity" could be defined, less formally, as "significant irregular contour in a particular section of a pattern."

Hoffman (1980) posited that some local features would be favored over others as discriminating features. The present research shows that variations in the complex sections of the contours of visual patterns are most utilized as discriminative stimuli. These results complement the findings of Goldmeire (1936/1972) and Kimchi and Palmer (1982), in that some local features play an important part in shape discrimination and others do not. Whereas these researchers pinpointed the size of the local features as a determinant of their role in shape discrimination, it now appears that complexity is also an important variable.

There must, of course, be some conditions under which subjects will learn to base their discriminatory responses on relatively simple local features in sections of contour. No knowledge of the results (NKR) was given to the subjects in the present experiment, nor was any overt reinforcement given. It has been shown many times for many types of patterns that discrimination ordinarily improves under NKR conditions (Bersted, Brown, \& Evans, 1969; Edmond, Evans, \& Mueller, 1966; Edmonds, Mueller, \& Evans, 1966; Evans \& Arnoult, 1967). On the other hand, knowledge of results (KR) has sometimes (but not always) been shown to lead to somewhat greater or more rapid learning (Brown, Walker, \& Evans, 1968). It is quite plausible that $\mathrm{KR}$, a reinforcement contingency, or some other change in paradigm could have produced a shift in discrimination from local features in complex contour sections to those in simple contour sections.

\section{REFERENCES}

Bersted, C. T., Brown, B. R., \& Evans, S. H. (1969). Free sorting with stimuli clustered in a multidimensional attribute space. Perception \& Psychophysics , 6, 409-413.

Brown, B. R., Walker, D. W., \& Evans, S. (1968). Schematic concept formation as a function of constraint redundancy and knowledge of results. Psychonomic Science, 11, 75-76.

Edmonds, E. M., Evans, S. H., \& Mueller, M. R. (1966). Learning how to learn schemata. Psychonomic Science, 6, 177-178.

Edmonds, E. M., Mueller, M. R., \& Evans, S. H. (1966). Effects of knowledge of results on mixed schema discrimination. Psychonomic Science, 6, 377-378.

Evans, S. H., \& ARNoult, M. D. (1967). Schematic concept formation: Demonstration in a free sorting task. Psychonomic Science, 9, 221-222.

FosTeR, D. H. (1978). Visual comparison of random-dot patterns: Evidence concerning a fixed visual association between features and feature-relations. Quarterly Journal of Experimental Psychology, 30, 637-654.

GIBSON, E. J. (1969). Principles of perceptual learning and development. New York: Appleton-Century-Crofts.

GoldmeIRE, E. (1972). Similarity in visually perceived forms. Psychological Issues, 8(1, Whole No. 29). (Originally published 1936)

HofFman, J. E. (1980). Interaction between global and local levels of form. Journal of Experimental Psychology: Human Perception \& Performance, 6, 222-234.

KImCHI, R., \& PALMER, S. E. (1982). Form and texture in hierarchically constructed patterns. Journal of Experimental Psychology: Human Perception \& Performance, 8, 521-535.

PASNAK, R. (1971). Pattern complexity and response to distinctive features. American Journal of Psychology, 84, 235-245.

PASNAK, R., \& TYER, Z. E. (1985). Distinctive local features of visual patterns Bulletin of the Psychonomic Society, 23, 113-115.

(Manuscript received for publication October 14, 1985.) 